\title{
EDITORIAL
}

\section{The Drop Tower Bremen}

\section{Dreyer}

Received: 22 September 2010 / Accepted: 22 September 2010 / Published online: 6 October 2010

(C) Springer Science+Business Media B.V. 2010

The drop tower Bremen was inaugurated on September 28,1990 . Then, it was not clear that such a facility, with $5 \mathrm{~s}$ free fall time of highly compensated gravity would turn out to be a great leap forward for research under space conditions.

Today, 20 years later, drop tower facilities all over the world play an important role. They serve all disciplines where gravity dependent phenomena play a role, in two ways: to prepare experiments with longer microgravity time on suborbital flights, retrievable capsules, the space shuttle and the international space station, or in many cases to provide sufficient data for comparison with theory and numerical simulations.

This topical issue on drop tower related research, on the occasion of the 20th anniversary of the drop tower Bremen presents actual results from different research fields: fluid mechanics, combustion, dust interaction, fundamental physics and space sciences. These examples are taken from more than 5,500 free fall and catapults tests (since 2004 allowing $9.3 \mathrm{~s}$ of compensated gravity), for more than 150 different test facilities with research teams from 40 nations that have used the drop tower since then.

The percentage of drop or catapult tests in the different fields are: combustion $33 \%$, space sciences $24 \%$, fundamental physics $22 \%$, fluid physics $11 \%$, biology $7 \%$, and material sciences $2 \%$. Sixty tests were used for testing of space technology related developments. A large number of scientific publications proves: research under short term microgravity is successful and will be a basis for future developments in this field.

M. Dreyer $(\varangle)$

Center of Applied Space Technology and Microgravity,

University of Bremen, Am Fallturm,

28359 Bremen, Germany

e-mail: dreyer@zarm.uni-bremen.de 